\title{
On a transformation between hierarchies of integrable equations
}

\author{
Metin Gürses a, Kostyantyn Zheltukhin ${ }^{\text {b,* }}$ \\ ${ }^{a}$ Department of Mathematics, Faculty of Sciences, Bilkent University, 06800 Ankara, Turkey \\ ${ }^{\mathrm{b}}$ Department of Mathematics, Faculty of Sciences, Middle East Technical University, 06531 Ankara, Turkey
}

Received 24 May 2005; received in revised form 17 October 2005; accepted 18 October 2005

Available online 24 October 2005

Communicated by A.P. Fordy

\begin{abstract}
A transformation between a hierarchy of integrable equations arising from the standard $R$-matrix construction on the algebra of differential operators and a hierarchy of integrable equations arising from a deformation of the standard $R$-matrix is given.

(C) 2005 Elsevier B.V. All rights reserved.
\end{abstract}

PACS: 02.30.Ik

Keywords: Integrable systems; Symmetries; Transformation

In a recent paper [1] a new hierarchy of integrable equations has been constructed through the deformation of a standard $R$-matrix on the algebra of pseudo-differential operators. We give a transformation between the hierarchy constructed in [1] and a hierarchy obtained through a standard $R$-matrix. The transformation is between corresponding vector fields (i.e. symmetries).

Let $\mathfrak{g}$ be the Lie algebra of pseudo-differential operators

$$
\mathfrak{g}=\left\{\sum_{i \in \mathbf{Z}} u_{i}(x) D^{i}\right\}
$$

with the commutator $\left[L_{1}, L_{2}\right]=L_{1} L_{2}-L_{2} L_{1}$. The algebra $\mathfrak{g}$ can be decomposed into Lie subalgebras $\mathfrak{g}_{\geqslant k}=\left\{\sum_{i \geqslant k} u_{i}(x) D^{i}\right\}$ and, $\mathfrak{g}_{i<k}=\left\{\sum_{i<k} u_{i}(x) D^{i}\right\}$ where $k=0,1,2$ (only for such $k$ one has Lie subalgebras). The standard $R$-matrix is given by $R_{k}=\frac{1}{2}\left(P_{\geqslant k}-P_{<k}\right)$, where $P_{\geqslant k}$ and $P_{<k}$ are projection operators on $\mathfrak{g}_{\geqslant k}$ and $\mathfrak{g}_{<k}$, respectively. The Lax hierarchy is

$$
L_{t_{n}}=\left[R\left(L^{n}\right), L\right]=\left[\left(L^{n}\right)_{\geqslant k}, L\right], \quad L \in \mathfrak{g}, n=1,2, \ldots
$$

The above equations involves infinitely many fields. To have a consistent closed equations with a finite number of fields we restrict the Lax operators as follows

$$
\begin{array}{ll}
k=0 & L_{0}=D^{N}+u_{N-2} D^{N-2}+\cdots+u_{1} D+u_{0} \\
k=1 & L_{1}=D^{N}+u_{N-1} D^{N-1}+\cdots+u_{0}+D^{-1} u_{-1} \\
k=2 & L_{2}=u_{N} D^{N}+u_{N-1} D^{N-1}+\cdots+D^{-1} u_{-1}+D^{-2} u_{-2} .
\end{array}
$$

See [2] for more details on the $R$-matrix formalism.

Recently in [1] the deformations of the above $R$-matrices were introduced. Most of the introduced deformed $R$-matrices do not lead to the new hierarchies. A new hierarchy is obtained through a deformation of $R$-matrix $R_{1}=\frac{1}{2}\left(P_{\geqslant 1}-P_{<1}\right)$. Let

\footnotetext{
* Corresponding author.

E-mail addresses: gurses@fen.bilkent.edu.tr (M. Gürses), zheltukh@metu.edu.tr (K. Zheltukhin).
} 
$P_{=i}(L)=(L)_{=i}$ denotes coefficient of $D^{i}$ in the expansion of $L \in \mathfrak{g}$. Then the deformed $R$-matrix is

$$
\tilde{R}=\frac{1}{2}\left(P_{\geqslant 1}-P_{<1}\right)+\varepsilon P_{=0}(\cdot) D,
$$

where $\varepsilon$ is a deformation parameter. The hierarchy is

$$
L_{t_{n}}=\left[\tilde{R}\left(L^{n}\right), L\right], \quad L \in \mathfrak{g}, n=1,2, \ldots .
$$

The above equations involves infinitely many fields, to have a consistent closed equation with finite number of fields we restrict the Lax operator as $\tilde{L}=u_{N} D^{N}+u_{N-1} D^{N-1}+\cdots+u_{0}+D^{-1} u_{-1}$. Then the new hierarchy is

$$
\tilde{L}_{t_{n}}=\left[\left(\tilde{L}^{n}\right)_{\geqslant 1}+\epsilon\left(\tilde{L}^{n}\right)_{=0} D, \tilde{L}\right], \quad n=1,2, \ldots,
$$

note that $\tilde{L}=\left.L_{2}\right|_{u_{-2}=0}$. See [1] for more details.

In this work we shall show that the new hierarchy (8) is related to the hierarchy corresponding to $R$-matrix $R_{2}$ with reduced Lax operator $\tilde{L}=\left.L_{2}\right|_{u_{-2}=0}$. So we relate hierarchy (8) to the hierarchy

$$
\tilde{L}_{t_{n}}=\left[\left(\tilde{L}^{n}\right)_{\geqslant 2}, \tilde{L}\right], \quad n=1,2, \ldots .
$$

We note that both hierarchies have the same Lax operator.

The construction of the transformation is based on expressing $\left(\tilde{L}^{n}\right)_{=1}$ and $\left(\tilde{L}^{n}\right)_{=0}$ in terms of coefficients of $\left[\left(\tilde{L}^{n}\right)_{\geqslant 2}, \tilde{L}\right]$, for $n \in \mathbf{N}$.

Proposition 1. Let $\tilde{L}=\left.L_{2}\right|_{u_{-2}=0}$, then

$$
\begin{aligned}
& \left(\left[\left(\tilde{L}^{n}\right)_{\geqslant 2}, \tilde{L}\right]\right)_{=N}=-\left(\left[\left(\tilde{L}^{n}\right)_{=1} D, \tilde{L}\right]\right)_{=N}, \\
& \left(\left[\left(\tilde{L}^{n}\right)_{\geqslant 1}, \tilde{L}\right]\right)_{=N-1}=-\left(\left[\left(\tilde{L}^{n}\right)_{=0}, \tilde{L}\right]\right)_{=N-1}
\end{aligned}
$$

for all $N(N$ is order of operator $\tilde{L})$.

Proof. Comparing powers of $D$ on the right- and left-hand side of the equality

$$
\left[\left(\tilde{L}^{n}\right)_{\geqslant 1}, \tilde{L}\right]=-\left[\left(\tilde{L}^{n}\right)_{<1}, \tilde{L}\right]
$$

we have

$$
\left(\left[\left(\tilde{L}^{n}\right)_{\geqslant 1}, \tilde{L}\right]\right)_{=N}=0 .
$$

Then

$$
\left(\left[\left(\tilde{L}^{n}\right)_{\geqslant 2}, \tilde{L}\right]\right)_{=N}=-\left(\left[\left(\tilde{L}^{n}\right)_{=1} D, \tilde{L}\right]\right)_{=N} .
$$

In the same way, comparing powers of $D$ on the right- and left-hand side of the equality

$$
\left[\left(\tilde{L}^{n}\right)_{\geqslant 0}, \tilde{L}\right]=-\left[\left(\tilde{L}^{n}\right)_{<0}, \tilde{L}\right]
$$

we have

$$
\left(\left[\left(\tilde{L}^{n}\right)_{\geqslant 0}, \tilde{L}\right]\right)_{=N-1}=0 .
$$

So,

$$
\left(\left[\left(\tilde{L}^{n}\right)_{\geqslant 1}, \tilde{L}\right]\right)_{=N-1}=-\left(\left[\left(\tilde{L}^{n}\right)_{=0}, \tilde{L}\right]\right)_{=N-1} .
$$

The above equalities $(10)$ and (11) allows us to express $\left(\tilde{L}^{n}\right)_{=1}$ and $\left(\tilde{L}^{n}\right)_{=0}$ in terms of coefficients of $\left[\left(\tilde{L}^{n}\right)_{\geqslant 2}, \tilde{L}\right]$ for all $N$.

Let us give an example for $N=1$.

Proposition 2. Consider the Lax operator $\tilde{L}=u D+v+D^{-1} w$. Let

$$
\left[\left(\tilde{L}^{n}\right)_{\geqslant 2}, \tilde{L}\right]=f_{n} D+g_{n}+D^{-1} h_{n},
$$

which gives the hierarchy (9) with the standard R-matrix and

$$
\left[\left(\tilde{L}^{n}\right)_{\geqslant 1}+\left(\tilde{L}^{n}\right)_{=0} D, \tilde{L}\right]=p_{n} D+q_{n}+D^{-1} r_{n},
$$


which gives the hierarchy (8) with the deformed $R$-matrix, $n=1,2, \ldots$ The coefficients $f_{n}, g_{n}, h_{n}, p_{n}, q_{n}, r_{n}$ are functions of $u$, $v, w$ and their derivatives. Then

$$
\left(p_{n}, q_{n}, r_{n}\right)^{T}=\mathcal{T}\left(f_{n}, g_{n}, h_{n}\right)^{T}
$$

where

$$
\mathcal{T}=\left(\begin{array}{ccc}
\varepsilon u_{x} D^{-1} v_{x} D^{-1} u^{-2}-\varepsilon u v_{x} D^{-1} u^{-2} & \varepsilon u_{x} D^{-1} u^{-1}-\varepsilon & 0 \\
u v_{x} D^{-1} u^{-2}+\varepsilon v_{x} D^{-1} v_{x} D^{-1} u^{-2} & 1+\varepsilon v_{x} D^{-1} u^{-1} & 0 \\
\left((u w)_{x}+\varepsilon w v_{x}\right) D^{-1} u^{-2}+\varepsilon w_{x} D^{-1} v_{x} D^{-1} u^{-2}+w u^{-1} & \varepsilon w u^{-1}+\varepsilon w_{x} D^{-1} u^{-1} & 1
\end{array}\right)
$$

Proof. Let $\left(\tilde{L}^{n}\right)_{=1}=A_{n}$ and $\left(\tilde{L}^{n}\right)_{=0}=B_{n}$. The equality (10) implies that

$$
f_{n}=-\left(\left[A_{n} D, \tilde{L}\right]\right)_{=1},
$$

hence, we can find

$$
A_{n}=u D^{-1} u^{-2} f_{n} .
$$

Using the equality (11) we have

$$
g_{n}+\left(\left[A_{n} D, \tilde{L}\right]\right)_{=0}=-\left(\left[B_{n}, \tilde{L}\right]\right)_{=0},
$$

hence, we can find

$$
B_{n}=D^{-1}\left(u^{-1} g_{n}+v_{x} D^{-1} u^{-2} f_{n}\right) .
$$

From the equality

$$
\left[\left(\tilde{L}^{n}\right)_{\geqslant 1}+\varepsilon\left(\tilde{L}^{n}\right)_{=0} D, \tilde{L}\right]=\left[\left(\tilde{L}^{n}\right)_{\geqslant 2}, \tilde{L}\right]+\left[\left(A_{n}+\varepsilon B_{n}\right) D, \tilde{L}\right]
$$

we can find the transformation between the vector fields

$$
\begin{aligned}
& p_{n}=u_{x} \varepsilon B_{n}-u \varepsilon B_{n, x}, \\
& q_{n}=g_{n}+v_{x}\left(A_{n}+\varepsilon B_{n}\right), \\
& r_{n}=h_{n}+\left(w\left(A_{n}+\varepsilon B_{n}\right)\right)_{x},
\end{aligned}
$$

where $A_{n}$ and $B_{n}$ are given by (22) and (24), respectively. Thus we obtain the transformation operator $\mathcal{T}$ in (20).

If we apply operator $\mathcal{T}$ to the simple symmetry $\left(u_{x}, v_{x}, w_{x}\right)^{T}$ we obtain $(0,0,0)^{T}$. Applying the operator $\mathcal{T}$ to $(0,0,0)^{T}$ we get

$$
\left(\begin{array}{c}
p_{1} \\
q_{1} \\
r_{1}
\end{array}\right)=\left(\begin{array}{c}
\varepsilon\left(v u_{x}-u v_{x}+u_{x}\right) \\
u v_{x}+\varepsilon\left(v v_{x}+v_{x}\right) \\
(u w)_{x}+\varepsilon(v w)_{x}+\varepsilon w_{x}
\end{array}\right) .
$$

This is the deformed system (8) for $n=1$ (with the inclusion of the symmetry $\left(u_{x}, v_{x}, w_{x}\right)^{T}$ ), [1]. If we take symmetry of the hierarchy (9) corresponding to $n=2$ (this is the reduced system [2,3])

$$
\left(\begin{array}{l}
f_{2} \\
g_{2} \\
h_{2}
\end{array}\right)=\left(\begin{array}{c}
u^{2} u_{x x}+2 u^{2} v_{x} \\
u^{2} v_{x x}+2 u(u w)_{x} \\
-\left(u^{2} w\right)_{x x}
\end{array}\right)
$$

and apply the operator $\mathcal{T}$ to this symmetry we obtain a second symmetry of the hierarchy (8)

$$
\left(\begin{array}{c}
p_{2} \\
q_{2} \\
r_{2}
\end{array}\right)=\left(\begin{array}{c}
\varepsilon u_{x} v^{2}-2 \varepsilon u v v_{x}-2 \varepsilon u^{2} w_{x}-\varepsilon u^{2} v_{x x} \\
2 u u_{x} w+2 u v v_{x}+2 u^{2} w_{x}+u u_{x} v_{x}+u^{2} v_{x x}+\varepsilon v^{2} v_{x}+2 \varepsilon u v_{x} w+\varepsilon u v_{x}^{2} \\
2 u_{x} v w+2 u v_{x} w+2 u v w_{x}-u_{x}^{2} w-3 u u_{x} w_{x}-u u_{x x} w-u^{2} w_{x x} \\
+2 \varepsilon u_{x} w^{2}+2 \varepsilon v v_{x} w+\varepsilon u_{x} v_{x} w+\varepsilon v^{2} w_{x}+4 \varepsilon u w w_{x}+\varepsilon u v_{x} w_{x}+\varepsilon u v_{x x} w
\end{array}\right) .
$$

Remark. In the example above we have constructed the transformation $\mathcal{T}$ for hierarchies with Lax operator of order one. In the same way we can construct the transformation between hierarchies with Lax operator of any order $N$. The operator $\mathcal{T}$ is not a recursion operator. It maps the symmetries of one system of evolution equations to symmetries of another system of evolution equations. 


\section{Acknowledgements}

This work is partially supported by the Turkish Academy of Sciences and by the Scientific and Technical Research Council of Turkey.

\section{References}

[1] B.M. Szablikowski, M. Blaszak, J. Math. Phys. 46 (2005) 042702.

[2] M. Blaszak, Multi-Hamiltonian Theory of Dynamical Systems, Texts and Monographs in Physics, Springer-Verlag, Berlin, 1998.

[3] M. Blaszak, Rep. Math. Phys. 48 (2001) 27. 